\title{
Voice Quality Analysis for Software Based Traffic Separation at the Access Layer of Hierarchical Design Model
}

\author{
Nixon Mtonyole \\ The University of Dodoma
}

\author{
Yona Andegelile \\ University of Dar es Salaam
}

\begin{abstract}
In many countries including Tanzania access network is still predominantly made up of the copper cable or point to point wireless connections. This has kept the network in large proportions passive, inflexible and relatively unreliable. Software based traffic separation at the access layer is a technique that is used to reduce the number of physical links to customer premises. This paper performs quality analysis for voice transmitted through such links (i.e. logical links) and it is observed that there is no major difference as to when dedicated physical links are used.
\end{abstract}

\section{General Terms}

Voice quality, Traffic separation, Hierarchical design model

\section{Keywords}

CPE, ISP, MPLS, PE and WiMAX

\section{INTRODUCTION}

Access Network is the subscriber part of the telecommunications network or the network connecting the subscribers to the Internet Service Providers (ISP). In many countries including Tanzania access network is still predominantly made up of the copper cable based or other point to point wireless connections. This has kept the network in large proportions passive, inflexible and relatively unreliable [1]. This traditional network has long been tailored to the services generally provided i.e. voice, leased lines, Internet, corporate data and video conference, sometimes each provided by separate equipment and networks.

At Service Providers core network, Virtual Private Networks (VPNs) are implemented to separate different services for different customers, and thus provide bandwidth per service demand. Still there is problem on the access part where separate point-to-point links are used to separate traffic.

Software based traffic separation is a traffic separation techniques which replaces the multiple hardware's approach of separating traffic at the access layer with a single hardware but traffic separated at software level, hence the name software based traffic separation. In this approach all traffic types are passed in the same physical link but logically separated (separated on software level). Each class of Traffic is being delivered to the customer premises with the same QoS guarantee (e. g bandwidth and delay) as when using separated physical links.

The study of the approaches used by most ISPs in Tanzania to deliver last mile shows that, still separate point to point links are in place. Different hardware's (antennas and other associated accessories) are needed to separate traffic to end customers [2]. With this, customers with need for branches data interconnection, branches voice interconnection and connection to the Internet need to have different Customer Premises Equipments (CPEs) for all these services. This approach does not only add cost to the customer and Internet Service Provider (ISP), but also introduces a number of disadvantages including difficulties for ISPs to support multiple services; safety risk to people working around, cause environmental pollution and also hinder network's scalability. Apart from that there raised difficulties when it comes to migration of corporate branches offices from one place to another whereby equipment for different services like antennas and associated accessories are needed to be shifted separately. Running and maintenance costs are high considering that, each equipment need to be maintained independently.

Software-defined networking (SDN) has gained a lot of attention in recent years, because it addresses the lack of programmability in existing networking architectures and enables easier and faster network innovation [3][4][5].

\section{BACKGROUND}

\subsection{OSI Layers Model}

The International Organization for Standardization (ISO) introduced the Open System Interconnect (OSI) standard in 1984, the system summarizing sophisticated network phenomena and cases on the seven layers. Layers in OSI are Physical layer (layer1), Data link layer (layer 2), Network layer (layer 3), Transport layer (layer 4), Session layer (layer 5), Presentation layer (layer 6) and Application layer (layer 7).

Each layer concerns network cases at identification phase. Each layer provides data for the next layer. Any software and hardware components are tackled on the related layer [6].

Logical separation of traffic at access layer is done by marking each traffic type. The traffic marking is done at OSI layer 2, based on IEEE802.1P. All other layers above don't need to know changes at this layer. With respect to layer 1, only single physical link will be used instead of multiple links [7][8][9].

\subsection{Cisco Three-Layer Hierarchical Model}

Cisco created its own three layers hierarchical model. This model is used to facilitate designing a scalable, reliable, costeffective hierarchical Internetwork. The Cisco three-layer hierarchical model refers to a conceptual guideline to follow in network design; it does not refer to the network data flow as the OSI model does. The three layers are Core, Distribution and Access (see Figure 1).

The access layer provides connectivity for the users. The distribution layer is used to forward traffic from one local network to another. Finally, the core layer represents a highspeed backbone layer between dispersed end networks. User traffic is initiated at the access layer and passes through the other layers if required. 


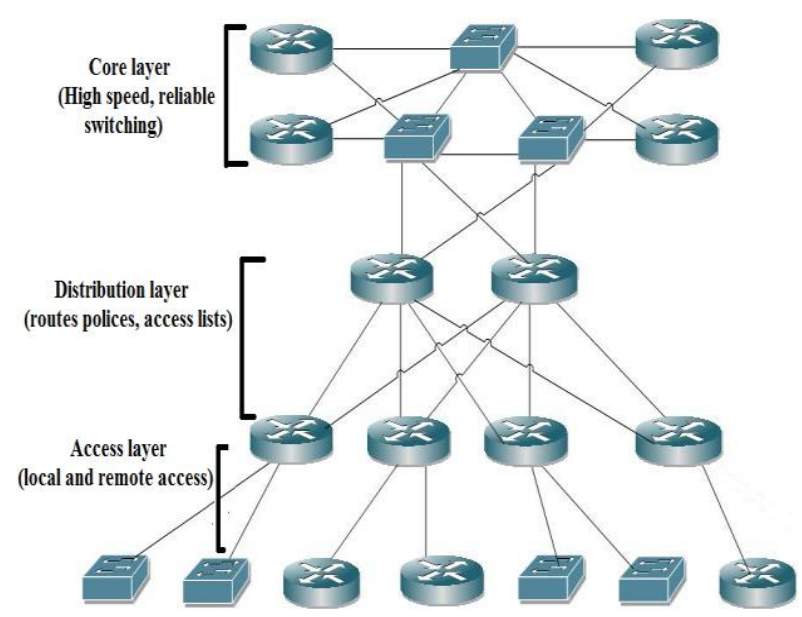

Fig 1: Three Layers Hierarchical Model

\subsection{VPN Implementation}

With high speed technological growth, Multiprotocol Label Switching (MPLS) has gained increasing interest from service providers over the past few years. It was originally used for traffic engineering purposes. Now, the latest application of MPLS is implementing provider provisioned VPNs. Using MPLS for implementing VPNs is a viable alternative to using a pure layer-2 solution, a pure layer-3 solution, or any of the tunneling methods commonly used for implementing VPNs [10]. When deciding on implementing an IP/MPLS-based VPN, the service provider has two choices, (i) A layer-3 approach, commonly referred to as MPLS Layer-3 VPNs and (ii) A layer-2 approach, commonly referred to as MPLS Layer-2 VPNs.

\subsection{WiMAX standard}

WiMAX is defined as Worldwide Interoperability for Microwave Access, which allows for broadband wireless access of information in the form of packet data. It was introduced in October 2001 and is also known as the IEEE 802.16 standard. The standard defines the air interface, also known as physical layer as well as MAC layer. These are bottom two layers, which defines the OSI 7 layer model that defines network technology. The protocols defined in these two layers allow it to control how packet data is handled at the air interface and at the first point of entry and exit of the system. The MAC layer(OSI layer 2) is designed in such a way that, it controls various physical specifications. The original specification allowed it to operate in the 10 to 60 $\mathrm{GHz}$ range. There were subsequent changes made to the standard, which curtailed it to the $2-11 \mathrm{GHz}$ range [11]. This was primarily done to improve its range. The standard is meant to operate in both licensed and unlicensed frequencies.

\subsection{6e WiMAX}

The 802.16d standard was released in 2004 and was set as the fixed version of the WiMAX standard. This version allows for Non Line of Sight (NLOS) connections and sets OFDM as the transmission protocol. The end user can use Fixed WiMAX from a fixed location or can move as a nomadic user usually moves at pedestrian speeds (see Figure 2). The fixed WiMAX standard is suitable for last mile connections and for backhaul connections to cellular networks and WiFi hotspots.

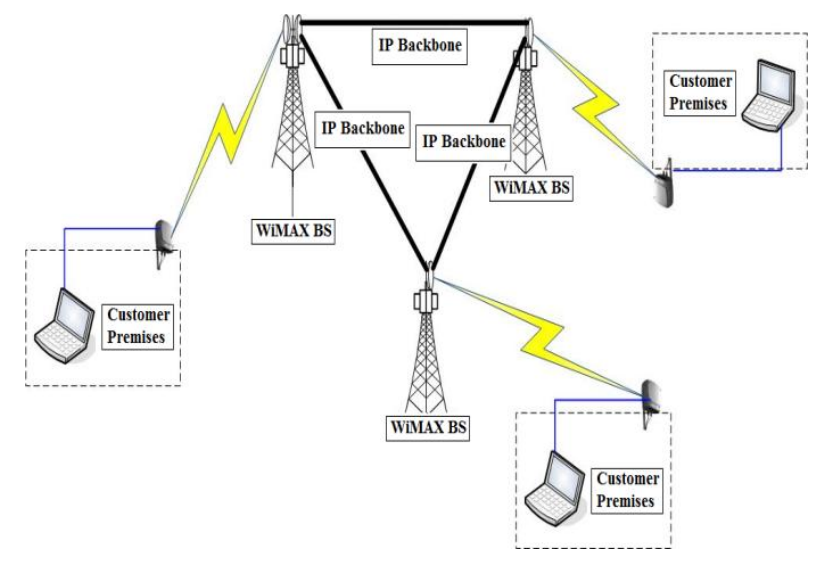

Fig 2: Fixed WiMAX network

Fixed WiMAX could be compared to the technologies like DSL and WiFi as last mile options. In the case of backhaul connections it could be compared to traditional wired backhaul solution like leased line and to wireless backhaul solution like satellite and microwave backhaul. Fixed WiMAX has however been superseded by the new $802.16 \mathrm{e}$ standard also known as mobile WiMAX.

\subsection{6e WiMAX}

The 802.16e WiMAX standard has been established to provide specifications for mobile broadband wireless access systems. The IEEE working group ratified the standard in December 2005. Although the fixed version of WiMAX focuses on the point to multipoint broadband access and last mile solution, the mobile version of WiMAX focus on mobility for broadband. The strength lies in the fact that as well as mobility, it can also support fixed and nomadic access. In fact, most $802.16 \mathrm{e}$ based networks begin operation as fixed networks and evolve into portable and then later mobile access (see Figure 3).

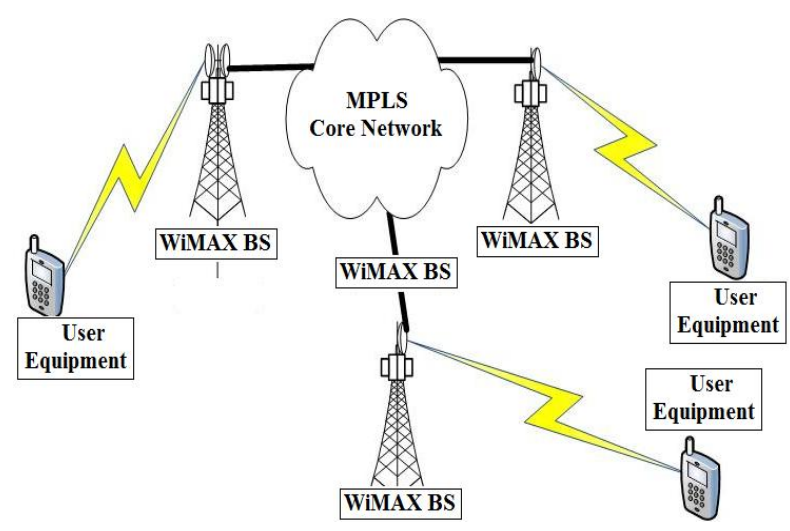

Fig 3: Mobile WiMAX network

\section{DESIGN AND IMPLEMENTATION OF SOFTWARE BASED TRAFFIC SEPARATION [12]}

\subsection{Logical Network Design}

MPLS backbone carries customer traffic from headquarter to branches and between branches. This is owned by service provider, and consists of $\mathrm{PE}$ routers at which customers are connected (see Figure 4). At the back bone, QoS is guaranteed for each traffic class according to Service Level Agreement (SLA) with a customer. Customer's branches are connected to 
nearest nodes. On PE edge router, each traffic type is given its own class, i.e. marked

$\mathrm{CE}$ is customer router located at customer area. At this router uplink traffic is marked according to traffic type. Between PE and $\mathrm{CE}$, one physical link is established, and logical links are created based on traffic class being carried by the link.

At customer site, there is a switch in which end equipments are connected. End equipment can be VoIP phone or computer for accessing Internet or data upload and download. A trunk port is created on the interface connecting to the $\mathrm{CE}$ router for the purpose of carrying all VLANs traffic. At WiMAX BS logical links are created within a single physical link, and each profile management is done per traffic type (i.e. each class)

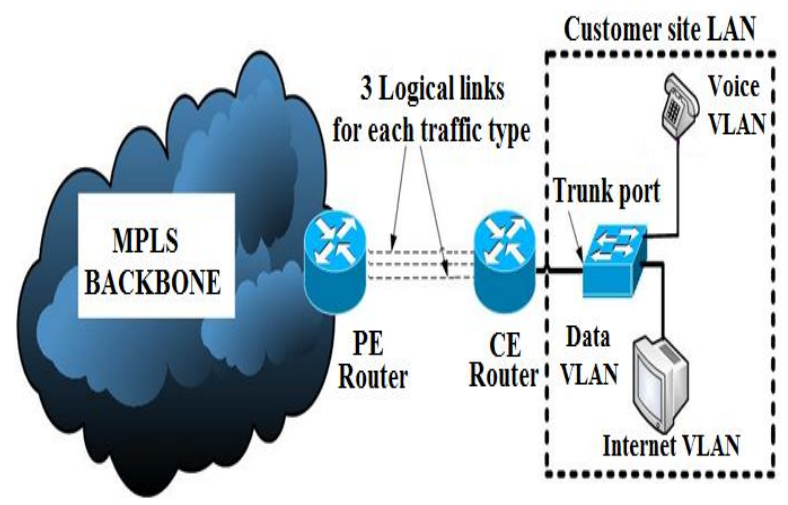

Fig 4: Logical Network Design [12]

\subsection{Physical Network Design}

Physically the network has PE edge router connected to WiMAX switch (2950 WiMAX switch) (see Figure 5). Switch has physical connection to Microwave transmitter (WIMAX Backhauls), which connect to WiMAX BS through Microwave link. Wireless link connect BS to CPE, which include ODU, IDU, and customer router and/or switch. 375001 and 3750-02 are switches which form part of provider network together with $7206-01$ and $72006-02$ provider edge routers.

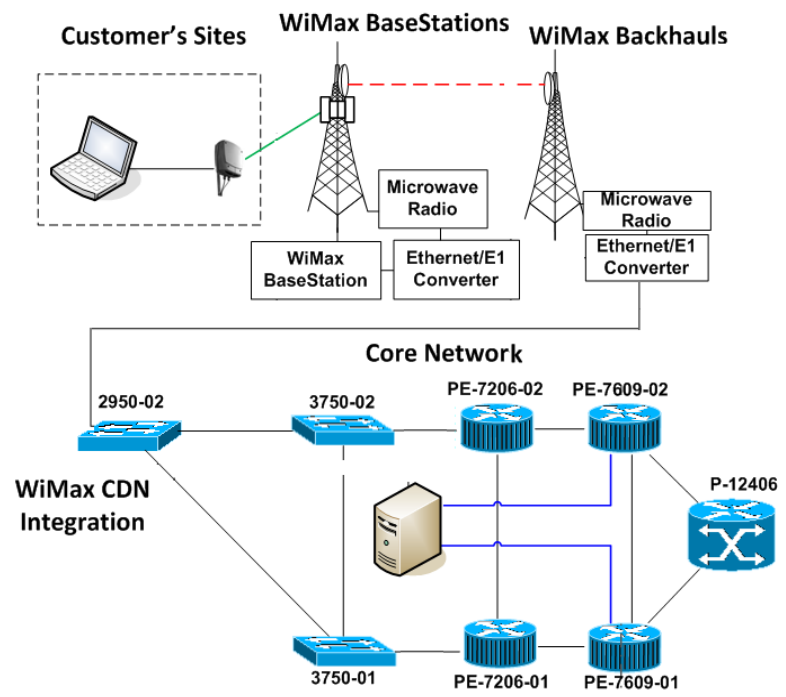

Fig 5: Physical Network Design

\subsection{Testing the Network Design}

The network which support multiservice was implemented as per design and thereafter some tests to verify its workability followed. Few tests cases were used to verify performance of the link before implementing multiservice over single physical channel then each test case was performed after combining into single physical channel where by specified KPIs were compared.

Voice has been tested for, Jitter, loss, Listening levels (clarity), Loudness Loss, delay in conversational and Talker echo Side tone. Calls were initiated from one asterisk client, processed by server and reach the other client (see Figure 6).

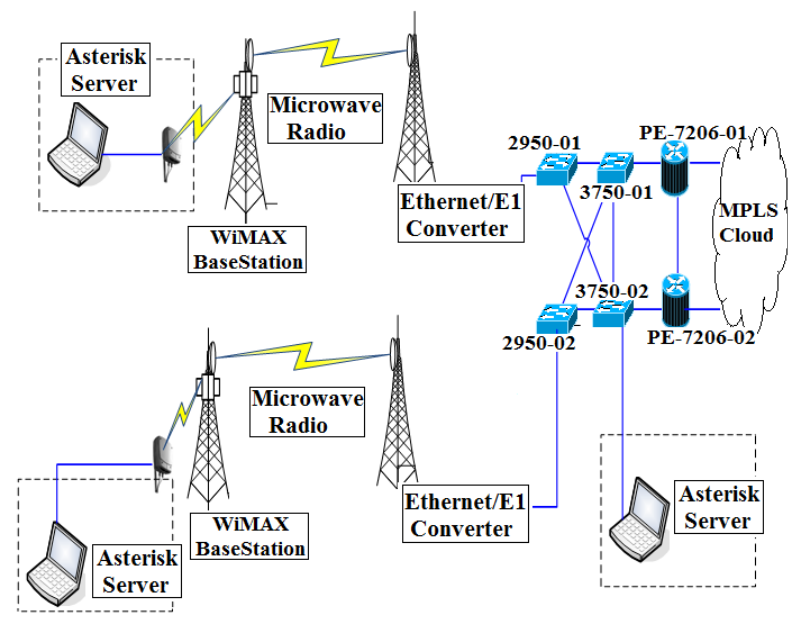

Fig 6: Set-up for Voice test case

\subsection{Implementation}

All PoPs (Point-of-Presence) in the core network are built in a very similar manner. Each PoP consists of two Cisco 7206 routers, connected to the Cisco 7609 routers. The 7609 router is connected to Cisco 12405 router which connects that POP with the ones in the other regions (see Figure 7). In each 7609 and 7206 routers there is another router of the same type, running Hot Standby Routing Protocol (HSRP) to provide redundancy in case of failure.

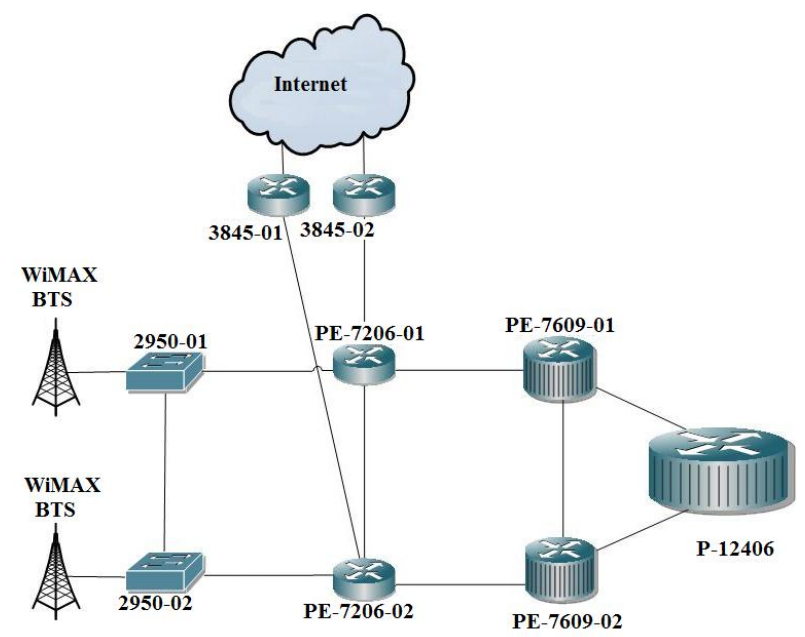

Fig 7: Site connection to Core Network and the Internet

In each region there is a Cisco 2950 switch connecting all WiMAX BS in the area. This switch is connected to 7206 router. Since network set for this work spread in one region, routing was done between WiMAX BS and PE router. 
Internet routers are connected to 7206 at Dar es Salaam site, and all other region connections are routed via this.

\subsubsection{WiMAX configuration}

At WiMAX BS, Convergence sub layer was configured in order to support profile management per COS (see Figure 8). Then site was created and registered, then radio link (physical) was created and configured, then, three profiles were created, one for voice with $128 \mathrm{kbps}$, one for Internet with $512 \mathrm{kbps}$ and one for Data.

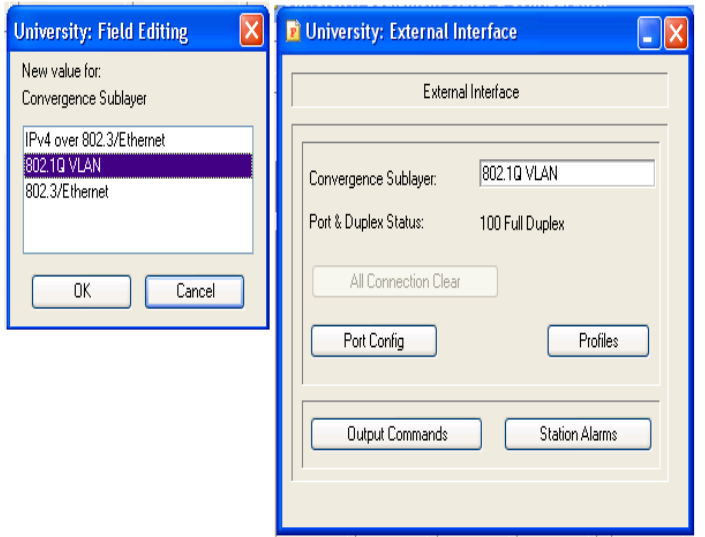

Fig 8: Configuration of Convergence Sub layer

Three types are available, IPV4 over Ethernet, 802.1Q which support profile management per COS, and Ethernet in which all traffic management are done at layer 2 .

Radio (physical) link has been configured with frequency parameter in which there are minimum, maximum and frequency shift (see Figure 9). These basically define radio link bandwidth. Others are power parameter, which define transmit power of the sector and radio parameters which define antenna gain and diversity.

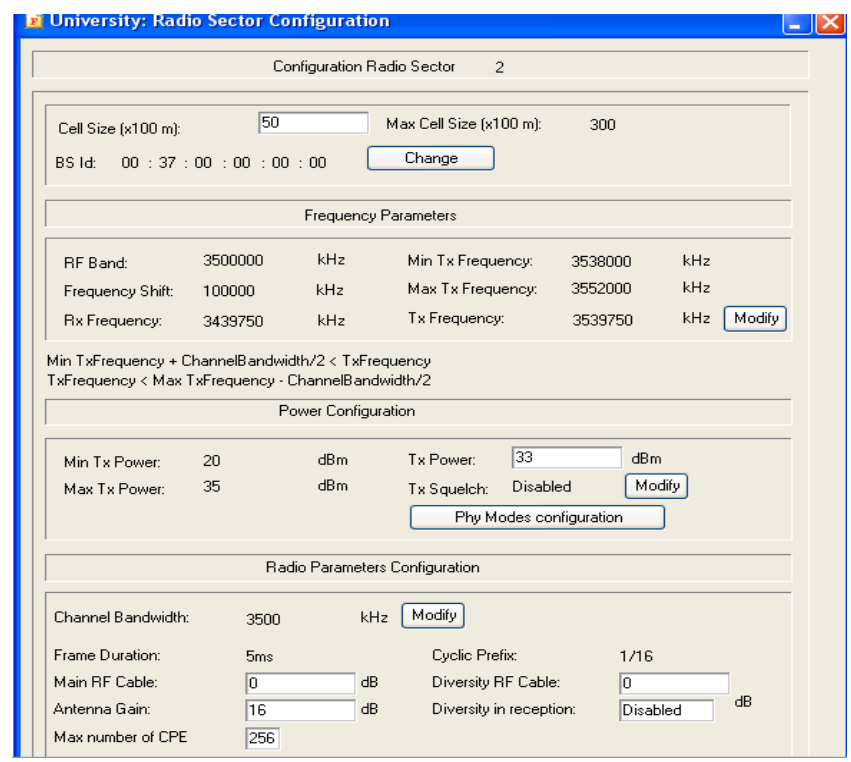

Fig 9: Radio Link Configuration

After creating Physical link, the BS was thereafter configured with modulation technique. This was configured separately for uplink and downlink (see Figure 10).

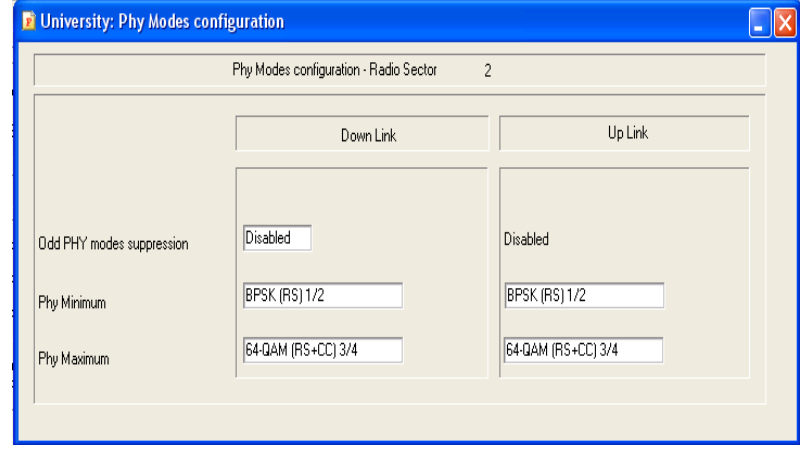

Fig 10: Modulation Method Configuration

The site has also been created or defines at WIMAX BS by specifying customer CPE and specific links to the site (see Figure 11).

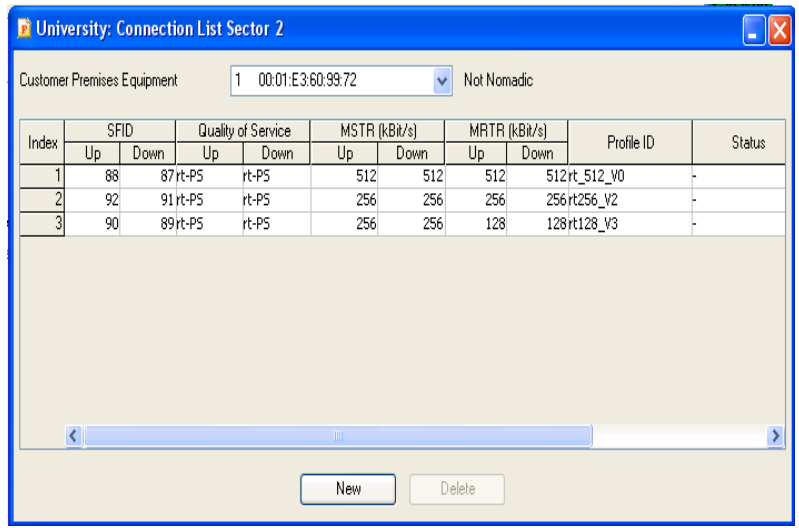

Fig 11: Test site creation

The site has been created with three logical links, each one identified by VLAN ID. Each logical link has been created with its own profile, where link parameters are configured. Internet link profile configuration (see Figure 12), data link profile configuration (see Figure 13) and Voice link profile configuration (see Figure 14).

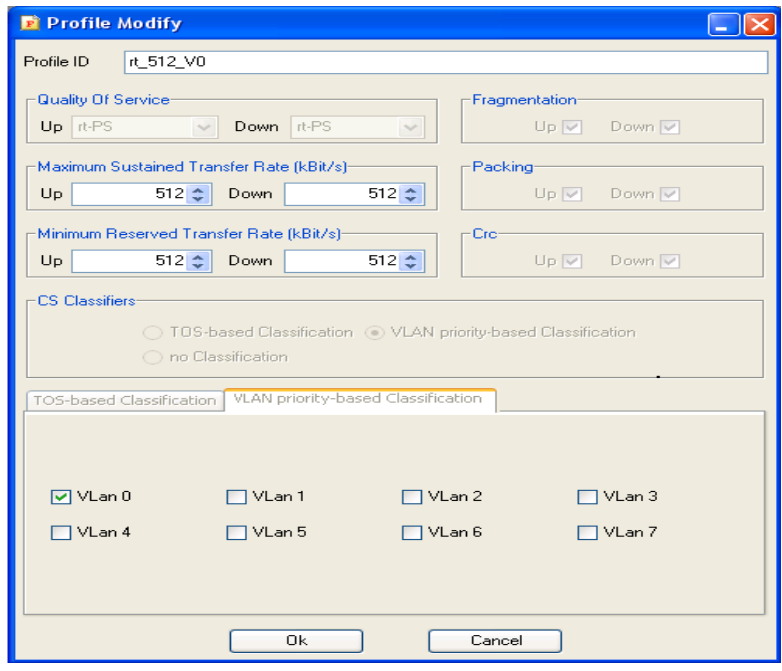

Fig 12: Internet Link Profile Configuration

This is Internet profile with $\operatorname{COS} 0$, and $512 \mathrm{kbps}$ bandwidth for both downlink and uplink. 


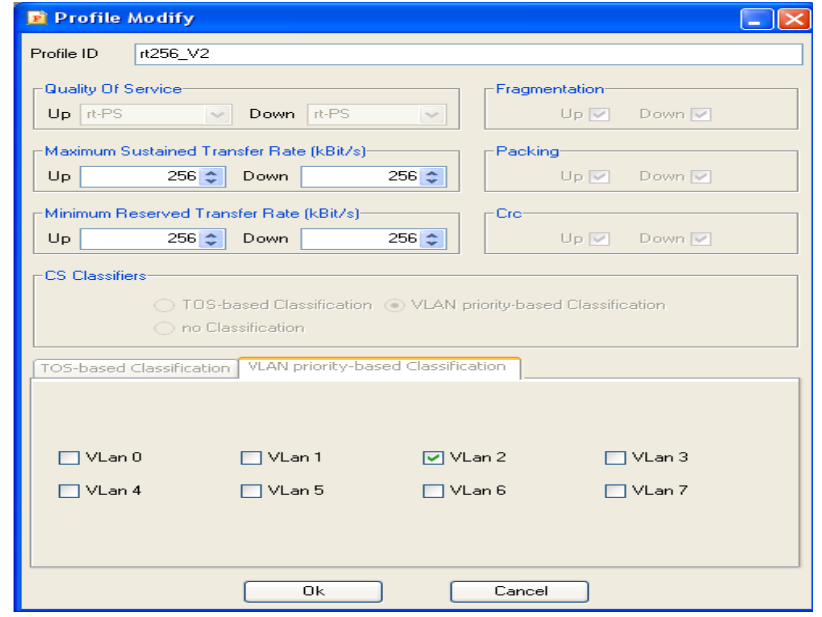

Fig 13: Data Link Profile Configuration

This is Data profile with COS 2, and $256 \mathrm{kbps}$ bandwidth for both uplink and downlink.

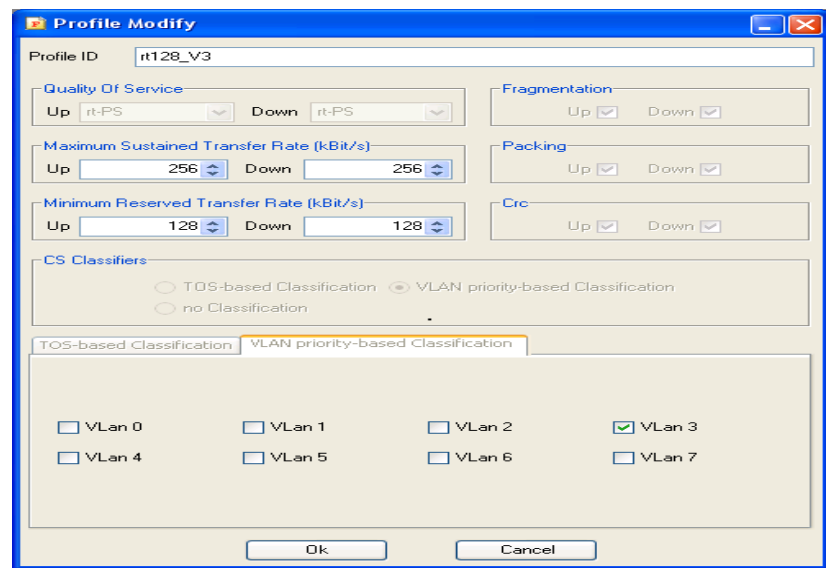

Fig 14: Voice Link Profile Configuration

This is VOIP profile with COS 3, and $128 \mathrm{kbps}$ bandwidth for both uplink and downlink.

\subsubsection{Test site configuration}

At the site, Cisco 1800 router was connected to Cisco 3750, and to WiMAX IDU, ODU was positioned in such a way that, clear line-of-site was maintained between BS and site antennas (see Figure 15).

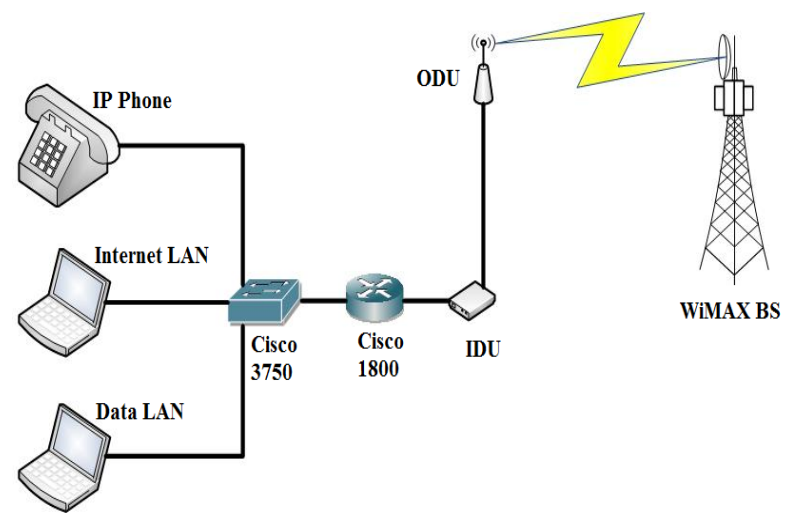

Fig 15: Test Site Connectivity to Base Station
A switch was configured with three VLANs, one for Data access, one for Internet access and another one for VoIP access. At the router, three VRFs were created for Data, Internet and VoIP.

\section{PERFORMANCE ANALYSIS 4.1 VoIP Traffic Link Performance Comparison}

Screen capture (see Figure 16), shows throughput experienced by end user when only Voice traffic was passing through the link before implementation. The average throughput on both transmit and receive was around $87 \mathrm{kbps}$. Note that: red bar charts represents download, green represents upload and yellow represents both download and upload.

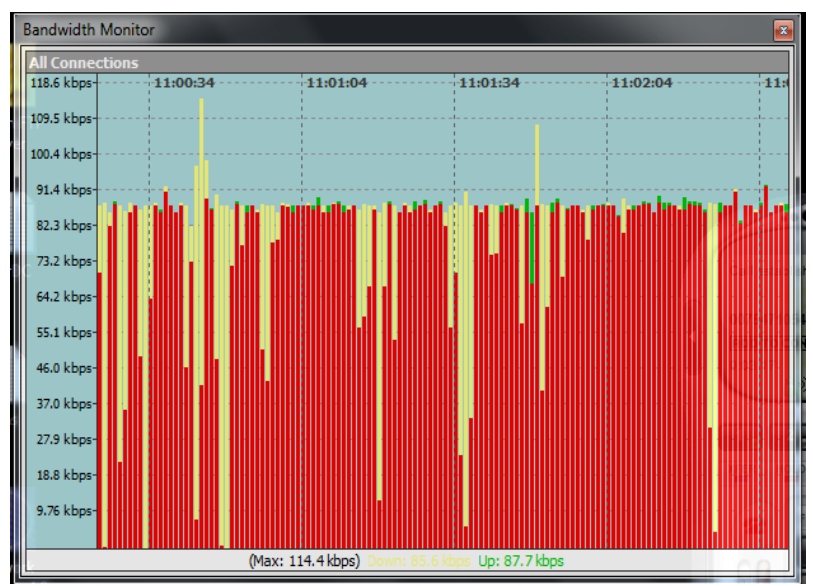

Fig 16: Voice Throughput before Implementation

Screen capture (see Figure 17), shows throughput experienced by end user when passing Voice traffic together with other traffic after implementation. The average throughput on both transmit and receive was around $86 \mathrm{kbps}$.

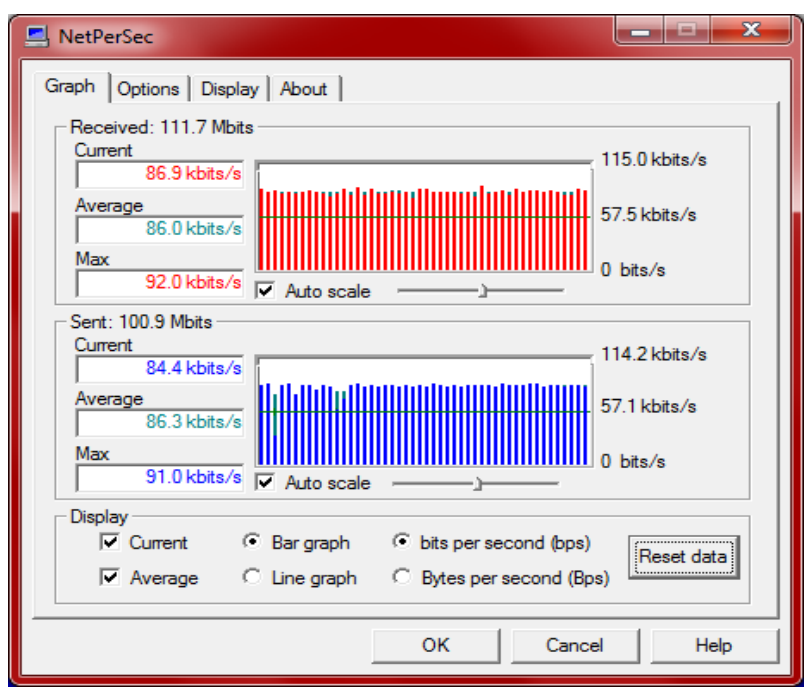

Fig 17: Voice Throughput after Implementation

Screen capture (see Figure 18), shows Round Trip Time taken by packet to travel from Asterisk client to Asterisk Server when only Voice traffic was passing through the link before implementation. The average value was around $141 \mathrm{~ms}$, min round trip time was $70 \mathrm{~ms}$, maximum was $1154 \mathrm{~ms}$, and no packet was lost out of 132 transmitted. 


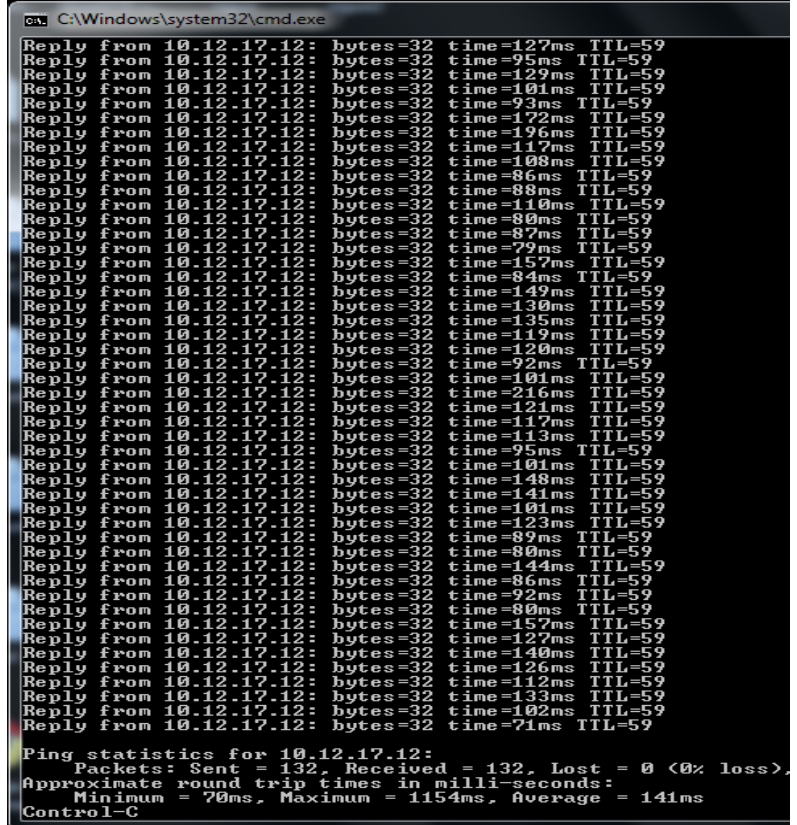

Fig 18: Voice RTT to asterisk server before implementation

Screen capture (see Figure 19), shows Round Trip Time taken by packet to travel from Asterisk client to Asterisk Server when Voice traffic was passing through the link together with other traffic types after implementation.

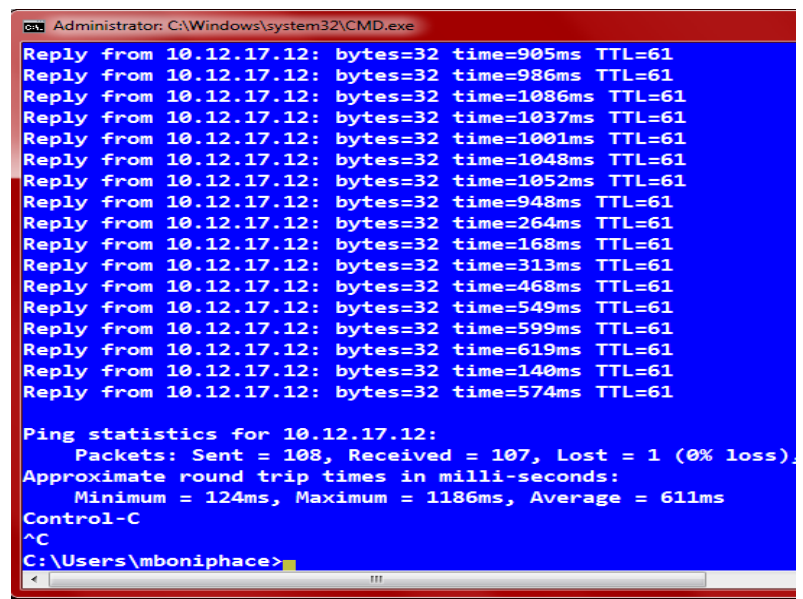

Fig 19: Voice RTT to asterisk server after implementation

The average round trip time to asterisk server was $611 \mathrm{~ms}, 1$ packet out of 108 was lost, maximum round trip time was $1186 \mathrm{~ms}$ and minimum was $124 \mathrm{~ms}$.

\subsection{Voice Quality}

The main challenges in designing a VoIP application are the quality of service (QoS) and the capacity of the gateways. Factors affecting the QoS are line noise, echo, the voice coder used, the talker overlap and the Jitter factor. The capacity of the gateway is related to the number of lines that can be supported in an enterprise environment.

The evaluation of speech quality is of critical importance in any VoIP application, mainly because quality is a key determinant of customer satisfaction. Traditionally, the only way to measure the perception of quality of a speech signal was through the use of subjective testing, i.e., a group of qualified listeners are asked to score the speech they just heard according to a scale from 1 to 5 . The most reliable method of speech quality assessment but it is highly unsuitable for online monitoring applications and is also very expensive and time consuming. Due to these reasons, models were developed to identify audible distortions through an objective process based on human perception. Objective methods can be implemented by computer programs and can be used in real time monitoring of speech quality. Algorithms for objective measurement of speech quality assessment have been implemented and the International Telecommunications Union has promulgated ITU-T P.862 standard, also known as Perceptual Evaluation of Speech Quality (PESQ), as its state of-the-art algorithm.

The tool used was Voice Quality (VQ) manager which monitors the voice conversation and plot the summary of the voice quality, results were as follows.

\subsubsection{Voice Quality before Implementation}

Screen capture (see Figure 20), shows the VoIP call trace from number 255754706511 to 255754711501 , when only voice traffic was passing through the link before implementation. The traces indicate the values of all metrics used to measure VoIP quality. The trace also indicates comment of each specific metric whether it is good, bad or satisfactory.

The following are the VoIP metrics feature that allows one to measure five metrics that reflect VoIP quality and their definitions.

Jitter: The variation in the delay of received packets in a flow. Latency: The time required for packets to travel from one Packet Shaper to another, Note that latency is calculated on the inbound interface only.

Packet loss: The percentage of lost packets.

R-Factor: VoIP transmission quality rating, with a typical range of 50-100. An R-Factor score is derived from multiple VoIP metrics, including latency, jitter, and loss.

MOS: The Mean Opinion Score, which provides a relative measurement of VoIP call quality. Packeteer measures MOS using a scale of 10-50. To convert to a standard MOS score (which uses a scale of 1-5), divide the Packeteer MOS value by 10 .

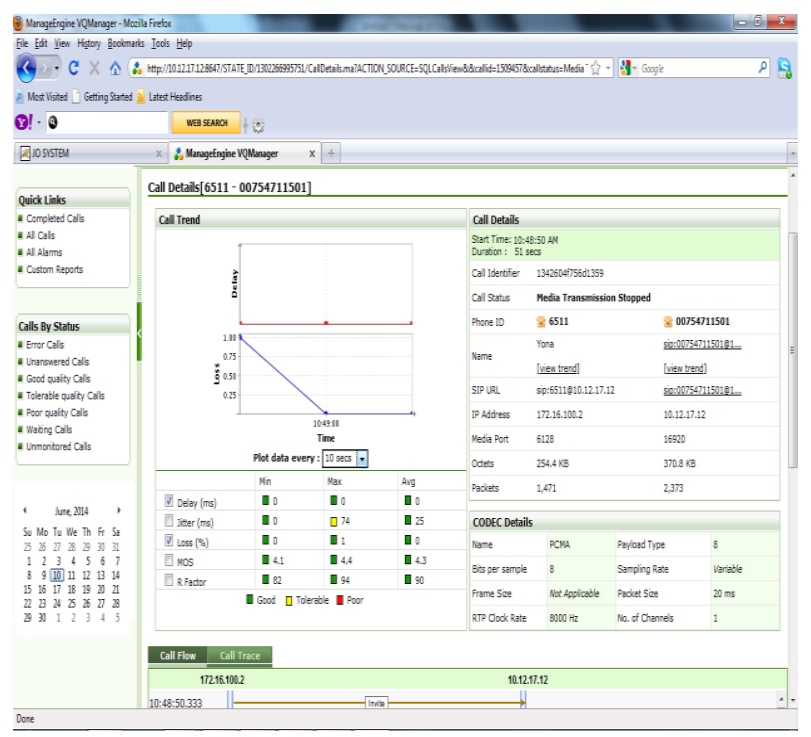

Fig 20: Voice Quality before Implementation 
From the trace (see Figure 20), Minimum jitter was 0 (good), maximum was 74 (Tolerable) and average was 25 (good). Minimum delay was 0 , maximum was 0 and average was 0 , all were good.

Packet loss; a maximum of 1 packet was lost, and an average of zero, this was good.

R-factor values, minimum was 82 , maximum was 92 and average was 90 , this was good.

MOS values; minimum value was 4.1 , maximum was 4.4 , and average was 4.3 , this was good.

\subsubsection{Voice Quality after Implementation}

Screen capture (see Figure 21), shows the VoIP call trace from number 255754706511 to 255754710453 , when voice traffic was passing through the link together with other traffic types after implementation. The traces indicate the values of all metrics used to measure VoIP quality. The trace also indicates comment of each specific metric whether it is good, bad or satisfactory.

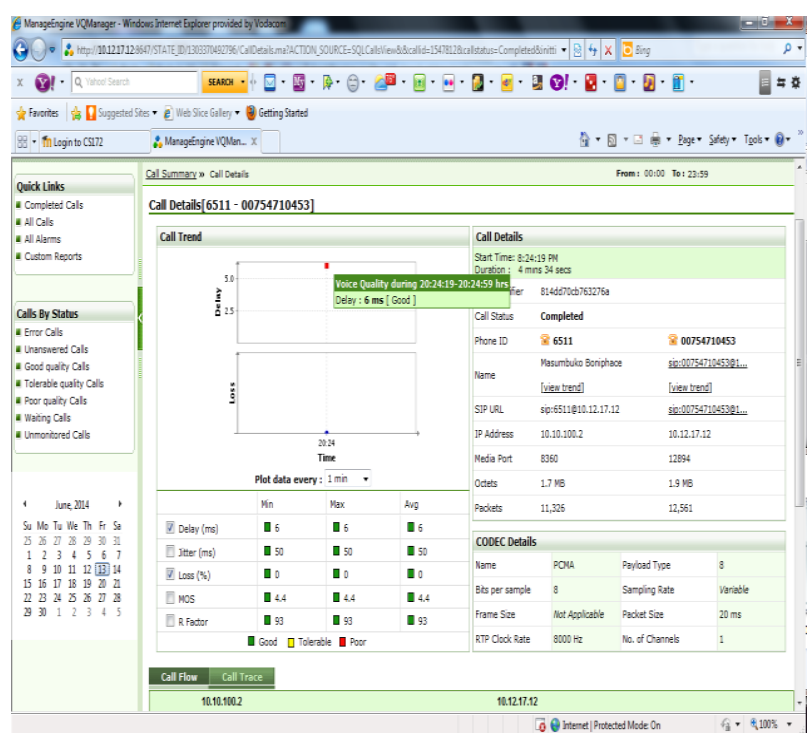

Fig 21: Voice Quality after Implementation

From the monitoring above,

Minimum jitter was 50 (good), maximum was 50(Tolerable) and average was 50;(good).

Minimum delay was 6 , maximum was 6 and average was 6 , all were good.

Packet loss; a maximum of 0 packet was lost, and an average of zero, this was good.

$\mathrm{R}$-factor values, minimum was 93, maximum was 93 and average was 93 , this was good.

MOS values; minimum value was 4.4 , maximum was 4.4 , and average was 4.4 , this was good.

\subsection{Node statistics}

Screen capture (see Figure 22), shows throughput as measured at the PE router voice logical interface connecting to the site after implementation. Green bar charts represents maximum download from user perspective, blue represents upload and corresponding lines shows traffic trend. Time periods between 7.00PM to 9.00PM represent time when traffic was captured. This was captured to demonstrate node behavior after implementation. Note that all interfaces are capable of carrying up to 1 Gigabits per second.

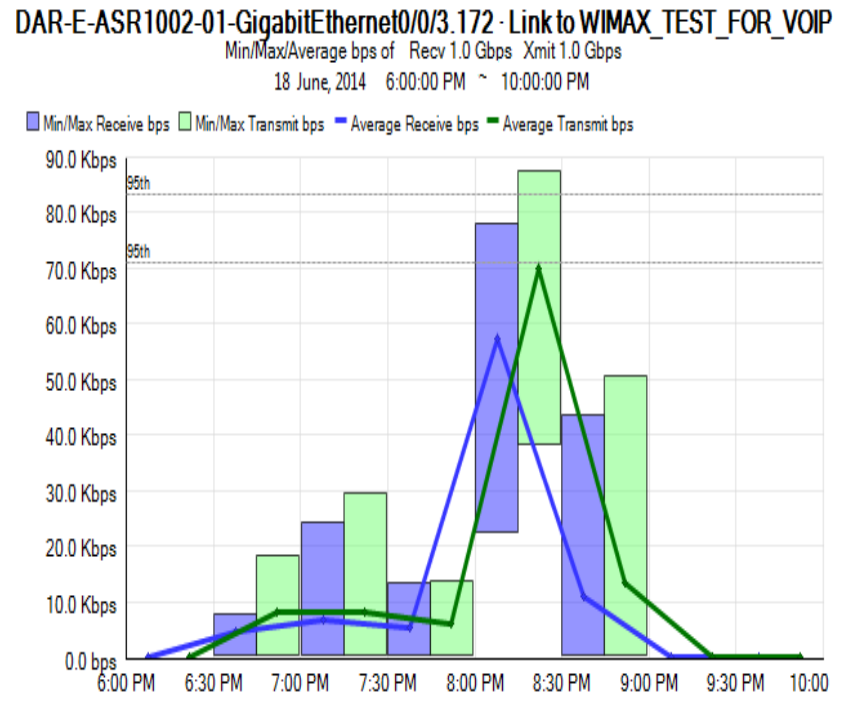

95th Percentile: Average Receive bps is $71.0061 \mathrm{Kbps}$, Average Transmit bps is 83.1375 Kbps SolarWinds Orion Core Services 2010.1

\section{Fig 22: PE router Voice Throughput Statistics}

Maximum for transmit was around $87 \mathrm{kbps}$ with average of 83 $\mathrm{kbps}$, for receive maximum was around 78 with average of $71.006 \mathrm{kbps}$. These values are reflected on user experience using bandwidth monitor and NetPerSec before and after implementation.

Screen capture (see Figure 23) shows volume transmitted as measured at the PE router voice logical interface connecting to the site after implementation.

$$
\begin{aligned}
& \text { DAR-E-ASR1002-01-GigabitEthernet0/0/3.172 - Link to WIMAX_TEST_FOR_VOIP } \\
& \text { Total Bytes Transferred Every } 30 \text { Minutes } \\
& \text { LAST } 24 \text { HOURS }
\end{aligned}
$$

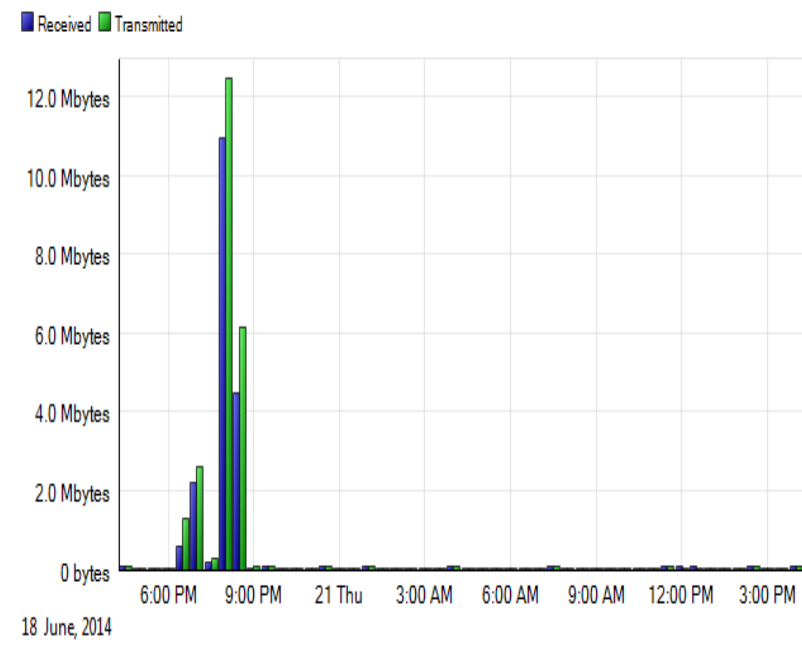

SolarWinds Orion Core Services 2010.1

\section{Fig 23: PE router Voice total bytes}

\section{CONCLUSION}

Converging services as a cheaper and scalable way to deliver ICTs services to end user has been a focus toward Next Generation Networks and is still a study where a lot of work has been done and is still being done. This paper looked at the voice quality when complexity in the last mile is removed by software based traffic separation and ISP deliver multiservice to a client through a single physical link, with the general idea being that this technology can give a new last mile 
implementation style, and that it's a cheaper technically viable solution compared to currently used methods.

Basic advantages accruing from such an implementation are, lowering implementation costs to both customers and providers, reducing complexity and costs in maintaining the network and reducing environmental pollution.

As the major concern is the quality of services, this study shows that there is no major difference on the quality of voice received when using dedicated physical link for voice and when using a shared link with other services. QoS and performance is almost the same.

\section{REFERENCES}

[1] Telia AB , Erricsson AB, Studentlitteratur AB, (2009) "Understanding Telecommunications".

[2] Yona Andegelile and Nixon Mtonyole, "Software based Traffic Separation at the Access Layer", International Journal of Engineering Research and Application, ISSN: 2248-9622, Vol. 4, Issue 7, July 2014, pp 158 - 162.

[3] Wolfgang Braun and Michael Menth, "Software-Defined Networking Using OpenFlow: Protocols, Applications and Architectural Design Choices" future internet ISSN 1999-5903 Published: 12 May 2014, pp 302-336

[4] Monsanto, C.; Reich, J.; Foster, N.; Rexford, J.; Walker, D., "Composing Software-Defined Networks", In Proceedings of the USENIX Syposium on Networked Systems Design \& Implementation (NSDI), Lombard, IL, USA, 2-5 April 2013; pp. 1-14.
[5] Astuto, B.N.; Mendonça, M.; Nguyen, X.N.; Obraczka, K.; Turletti, T. "A Survey of Software-Defined Networking: Past, Present, and Future of Programmable Networks", IEEE Commun. Surv. Tutor. 2014, doi:10.1109/SURV.2014.012214.00180.

[6] Rudenko, I. (2000), Cisco Routers, Coriolis Press, USA.

[7] Patra, S. Nedevschi, S. Surana, A. Sheth, L. Subramanian, and E. Brewer. Wildnet (2007), Design and implementation of high performance wifi based long distance networks. In NSDI'07, pages 87.

[8] Diane, T. (1999), Designing Cisco Networks, Cisco Press, USA.

[9] Giles, R. (1999), All-in-one CCIE Study Guide, McGraw Hill Press, USA.

[10] Martini, L.(2006), Transport of layer 2 frames over MPLS, IETF draft, draft-martini-12circuit-trans-mpls08.txt.

[11] Intel Corporate White Paper (2004), Wi-Fi and WiMAX Solutions, "Understanding Wi-Fi and WiMAX as MetroAccess Solutions,".

[12] Yona Andegelile and Nixon Mtonyole, "Software based Traffic Separation at the Access Layer", International Journal of Engineering Research and Application, ISSN: 2248-9622, Vol. 4, Issue 7, July 2014, pp158 - 162. 